\title{
Quantum-informational Principles for Physics
}

\section{Giacomo Mauro D'Ariano}

It is time to to take a pause of reflection on the general foundations of physics, re-examining the solidity of the most basic principles, as the relativity and the equivalence principles that are currently under dispute for violations at the Planck scale. A constructive criticism engages us in seeking new general principles, which reduce to the old ones as approximations holding in the physical domain already explored. At the very basis of physics are epistemological and operational rules for the same formulability of the physical law and for the computability of its theoretical predictions, rules that give rise to new solid principles. These rules lead us to a quantum-information theoretic formulation, hinging on a logical identification of the experimental protocol with the quantum algorithm.

The information-theoretic program for physics foundations has already been advocated in the past by several authors [ [U]. Recently the program succeeded in deriving the full structure of quantum theory from information-theoretic principles [, [1, 田, 回]: we will very briefly examine such principles in the following, as exemplars of good principles. The problem now is to extend the program to relativistic quantum field theory, the most fundamental theoretical structure of physics. The proposal suggested here is to ground quantum field theory on two new principles pertaining only formulability and computability of the physical law: 1) the Deutsch-Church-Turing principle, and 2) the topological homogeneity of interactions. As we will see, in conjunction with the principles of quantum theory, these two new principles entail a quantum cellular automata extension of quantum field theory.

The quantum automaton extends field theory in the sense that it includes localized states and measurements, for whose description quantum field theory is largely inadequate. The quantum automaton doesn't suffer any formal violation of causality, e.g. superluminal tails of the probability distributions. It is not afflicted by any kind of divergence, being exactly computable in-principle. Relativistic covariance and other symmetries are violated, but are recovered in the Fermi-scale thermodynamic limit.

The generality of the new principles does not deplete them of physical content. On the contrary, the Dirac automaton-the most elementary theory of this kind-despite its simplicity leads to unexpected interesting predictions, e.g. it anticipates a maximum mass for the Dirac particle just as a consequence of the unitariety of quantum evolution, without invoking black-hole general-relativity arguments. It also opens totally unexpected routes for redefining mechanical notions. As regards gravity, the theory seems to suggest the route of the emergent thermodynamic force of Jacobson-Verlinde [ [G, $\boldsymbol{\square}]$, here specifically as a purely quantum-digital effect of the Dirac automaton. 


\section{Good and bad principles}

Which principles are good and which are bad? We can limit ourself to four main different types of principles: 1) dogmatic, 2) empirical, 3) simplifying (or conventional), and 4) epistemological.

The dogma. This is definitely the worst case. Do we have dogmas in physics? I think that we have some, though subtle ones. Is it a blasphemy to regard the non existence of an absolute reference frame as a dogma? What about the reference frame of the background radiation? We indeed often invoke the frame of "fixed stars" for establishing an inertial frame. I think that denying the existence of an absolute is a relic of the repudiation of the anthropocentrism that followed the Keplerian revolution. We will come back to this dogma later.

The empirical principle. A principle is empirical if it has no logical motivation other than its empirical evidence. A typical example is the equivalence principle: the identity of the inertial mass with the gravitational mass is a fact, but do we have any good reason for it? The principle implies that the trajectory of a mass in a gravitational field is independent on the mass, and this leads us to reinterpret gravity as a property of space-the starting point of general relativity. But this is a re-interpretation of the principle, not a motivation. Another relevant example of empirical principle is the invariance of the speed of light with the reference system-quite an odd one, isn't it? This lead Einstein to his first formulation of special relativity. But the principle was later recognized to be just an instance of the more general Galilei principle (the invariance of the physical law with the reference frame) upon including the laws of electromagnetism, and this was definitely a great logical improvement. Empirical principles are definitely good temporary practical ones, as long as we relinquish further explanation.

The simplifying principle. A simplifying principle is an unverifiable conventional assumption that simplifies the formulation of the physical law. An example is the assumption of homogeneity of time (it is impossible to compare two different time-intervals in temporal sequence). But a purported non-homogeneity of time would introduce an unnecessary functional time-parameterization in the physical law. Another example is the assumption that the speed of light is isotropic in space. Reichenbach [8] correctly argued that in order to determine simultaneity of distant events we need to know the light speed, but in order to measure the light speed we need to establish simultaneity of two different events for for synchronizing clocks, leading to a logical loop. What we can do? Using a single clock we can only determine the two-way average speed of light on a closed path. Reichenbach wrote indeed unconventional Lorentz transformations for nonisotropic light speed, with the only result of introducing an additional anisotropy parameter that is totally irrelevant in practice. In conclusion: the simplifying principles are good ones, but we must keep in mind their conventional nature. 
The epistemological principle. This is the principle that I consider the most solid one: a principle that cannot be violated, even in-principle, because its violation will involve contradicting a logical argument. Somebody would argue that claiming principles only of this kind is equivalent to claiming an "ultimate theory of everything". True. But should this be a good reason for not seeking principles of this kind? And for evaluating their ultimate logical consequences? Clearly, to be a principle for physics it cannot involve only pure logic: it must also incorporate the basic axiomatic of the physical experiment. G. Ludwig has been a great advocate of such kind of principles [ [0]. Einstein himself formulated special relativity in terms of precise protocols for synchronizing clocks in order to establish coordinate systems. In the recent literature operational axiomatic frameworks of this kind have emerged for quantum theory, later converging to a

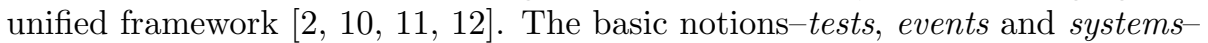
make the framework equivalent to a category theory for physics [ㅍ]. At the same time, it is also the skeleton axiomatization of a general information theory, and, as mentioned, it ultimately lead to the informational axiomatization of quantum theory $[\boldsymbol{\nabla}, \mathbf{[}, \mathbf{Q}, \mathbf{Q}, \mathbf{0}]$. A remarkable fact about the operational approach is that it logically identifies the experimental protocol with the computer algorithm, providing a stronger logical connection between theory and experiment.

\section{The relativity principle}

The relativity principle of Galilei and Einstein seems to possess a definite epistemological character, since it establishes the independence of the physical law from the reference system, apparently a necessary requirement for the law formulation and experimentation. The principle instead is based on the no-absolute dogma, since nothing forbids defining the law within an absolute frame and translating it to any other frame (what we do when we invoke the "fixed stars" frame). This viewpoint may look as a sacrilege, but we have no way out to violations of Lorentz covariance.

\section{The causality principle}

Causality has been always a taboo in physics. As a principle it is underlying all modern physics, and has been central to debates on the foundations of relativity and quantum mechanics for over a century. Despite this, there as been a philosophical train of thought arguing that this notion should be removed from physics, B. Russell being one of the major advocates of this opinion [4]]. Causality is such a natural assumption that is often overlooked as an axiom (see e.g. the first quantum axiomatization work of Hardy [四]). It is instead the first of the informational axioms of quantum theory $[\boldsymbol{\nabla}]$, also referred to as no signaling from the future. In simple words it says: in a cascade of measurements on the same system, the outcome probability of a measurement does not depend on the choice of the measurement performed at the output. The principle also implies the no-signaling without interaction, generally simply called "no-signaling", and also commonly known as "Einstein causality". I should make now clear that, 
being causality an axiom of quantum theory, any information purportedly originated in the future, as a time travel, would logically constitute a falsification of the theory. For example, it would mean to require nonlinearities in state evolution, or other variations of the theory.

As we will see later, in the present informational context special relativity emerges as an approximate principle due to the joint implication of three principles: 1) the causality principle, 2) the Deutsch-Church-Turing principle, and 3) the principle of topological homogeneity of interactions.

The problem of physical causation is a huge topic in philosophy, and a thorough discussion would take a thick volume. For the philosopher disbeliever I just want to add that the reconciliation with the Humean position (that causality is just a human way of looking at phenomena) passes through the probabilistic nature of the causal link stated in the axiom, which involve the comparison between two probabilities, whence the Humean viewpoint corresponds to the Bayesian interpretation of probability [प]. However, if causality cannot be proved, it can be falsified, as any other scientific theory. How? Just considering any binary test that is granted to be deterministic, namely to have zero probability for one outcome: if operating at the output of the test we can make this same outcome to happen, then we can logically claim a signaling from the future, given for granted the apparatus and its preparation.

Causal reasoning has always been a basic methodology in physics and in science generally, but the romantic dream of a time travel keeps alive the sentiment against it.

\section{Informational principles for quantum theory}

In addition to causality, there are five other informational principles that are needed for deriving quantum theory [छ]: ii) local tomography, iii) perfect distinguishability, iv) atomicity of composition, v) ideal compressibility, and vi) purification. All six principles apart from vi) hold for both classical and quantum information, and only the purification one singles out quantum theory.

The information-theoretical framework hinges around the notion of event, which can occur probabilistically and has inputs and outputs systems. A complete collection of such events with overall unit probability is what is called test-physically a measurement instrument. The systems are just the usual physical systems. Informationally, tests and events represent subroutines, whereas the systems are registers on which information is read and written. Axiom ii) (stating that joint states of multiple systems can be discriminated by measurements on single systems) has become now popular [[W], since it reconciles the holism of quantum theory with the reductionism of the experimental approach [4]]. Axiom iii) is crucial for hypothesis falsification, and reconciles probabilism with logic. Axiom iv) establishes that maximal knowledge of the episodes implies maximal knowledge of the full history, a requirement that seems obvious indeed. The compression axiom v) is the one that leads to the notion of subsystems (e.g. the qubit is a subsystem of the qutrit). Finally, the purification postulate vi) informally speaking is the principle of "conservation of informa- 
tion". In simple words it says that irreversibility and mixing can be always regarded as the result of discarding an environment, otherwise everything being describable in terms of pure states and reversible transformations. Another informal way of stating the principle is that ignorance about a part is always compatible with the maximal knowledge about the whole.

The six principles for quantum theory have nothing of "mechanical" nature: what I call "quantum theory" is just the abstract "theory of systems", i.e. the mathematical framework of Hilbert spaces, algebra of observables, unitary transformations, and has no bearing on the "mechanics" i.e. particles, dynamics, quantization rules, for which the name "quantum mechanics" would be more appropriate. Quantum mechanics, however, is just a small portion of the more general quantum field theory, which itself is a theory of systems: the quantum fields. The only mechanical elements remaining in quantum field theory are the so-called "quantization rules" (or the path-integral) that one may want to avoid in order to make the theory completely autonomous from the classical theory, whereas, reversely, it should be classical mechanics to be derived as an approximation of quantum field theory via a "classicalization" rule. But, how can we formulate a field theory that is quantum ab initio? We need more informational principles, in addition to the six ones of quantum theory. Those principles, which will substitute the relativity principles, are: the Deutsch-Church-Turing principle, and the principle of topological homogeneity.

\section{Substitutes for the relativity principle}

The Deutsch-Church-Turing principle. Rephrasing D. Deutsch [पष]: "Every physical process describable in finite terms must be perfectly simulated by a quantum computer made with a finite number of qubits and a finite number of gates". In the logic of specularity between experimental protocols and algorithms (both include also outcomes), I would say: Every finite experimental protocol is perfectly simulated by a finite quantum algorithm. It is immediate to see that the principle implies two sub-principles: a) the density of information is finite, and b) interactions are local. The kind of information that we are considering here is quantum, whence the assertion that the density of information is finite means that the dimension of the Hilbert space is finite. This means that e.g. the boson should be regarded as an asymptotic approximate notion. Richard Feynman himself is reported to like the idea of finite information density, because he felt that "There might be something wrong with the old concept of continuous functions. How could there possibly be an infinite amount of information in any finite volume?" [U]. The finite dimension of the Hilbert space also implies locality of interactions, namely that the number of quantum systems connected to each gate is finite.

Topological homogeneity of interactions. The principle states that the quantum algorithm describing a physical law is a periodic quantum network. In the informational paradigm the physical law is represented by a finite set of connected quantum gates, corresponding to a finite protocol, theoretically 
specular of a finite quantum algorithm. Thus locality of interactions is required in order to define a physical law in terms of a finite protocol under the local control of the experimenter, whereas homogeneity represents the universality of the law, which is assumed to hold everywhere and ever. It follows that algorithmically the physical law is represented by a quantum unitary cellular automaton of Werner and Schumacher [प्]]. The "space"-period and the "time"period of the automaton correspond to the minimum space and time units $l_{P}$ and $t_{P}$-the Planck distance and the Planck time, respectively. At some very small scale-the Planck scale-the world is discrete!

\section{The quantum cellular automaton}

Causality together with the Deutsch-Church-Turing principle imply that information propagates at finite speed, the maximum speed being the "speed of light" $c=l_{P} / t_{P}$-the causal speed of the automaton. The two principles together thus imply that the state of any finite set of systems can be evaluated exactly as the evolution for of finite number of time-steps of a larger but still finite number of systems in the past causal cone, regardless the quantum network being unbounded. As long as we evaluate evolutions for finite number of steps, we do not need to worry about boundary conditions. We take as vacuum state any state that is locally invariant under the automaton evolution. The localized states are then those that differ from the vacuum only for a finite number of systems. The future causal cone of these state-supporting systems is then the place where only we need to evaluate the evolution, again with no need of boundary conditions. We do not have any divergence, nor ultraviolet (no continuum), nor infrared (no calculation for infinite extension): the Deutsch-Church-Turing principle excludes tout court the continuum and the infinite dimension.

Recovering the old quantum field theory. The old field theory is recovered as an approximation via an analytical asymptotic evaluation of the automaton evolution in the Fermi-scale thermodynamic limit of infinitely many steps and delocalized states, the latter corresponding to customary quantum particles. In this way we obtain back e.g. the usual Dirac equation in the relativistic regime of small masses and momenta, but at the same time we also describe the physics of very large Planckian masses and ultrarelativistic huge momenta [एव]].

Emerging physics. It must be stressed that the homogeneity of interactions is a purely topological property, not a metrical one, the interactions making a network of connected systems where the length of each link of the graph has no physical meaning. The idea is that the space-time metric emerges from the pure topology by event counting, and the Planck length $l_{P}$ and time $t_{P}$ conceptually are just digital-analog conversion factors. Also the particle mass $m$ of the Dirac automaton is a pure number $0 \leq m \leq 1$, and the Planck mass $m_{P}$ is the conversion factor to customary kilograms. 
Universal automata constants. The three quantities $l_{P}, t_{P}, m_{P}$ are the irreducible universal constants of the automata theory, and the adimensional mass is the only parameter characterizing the Dirac automaton. The Planck constant can be now rewritten in terms of the automata universal constants as $\hbar=m_{P} l_{P}^{2} t_{P}^{-1}$.

Inertial mass. As I already explained in my previous FQXi essay [ $[2]$, the inertial mass is reinterpreted as the slowing down of the information flow via the coupling between the modes flowing along the directions in the network at maximal speed $c$ (for $d>1$ space-dimensions is a coupling between different chiralities [ए]]).

\section{Particle speed and Planck} mass as bound on mass. The speed of a zero-mass particle depends on the wavelength, and approaches zero at Planckian wavelengths anisotropically in space (see Fig. Ш). For massive particles the

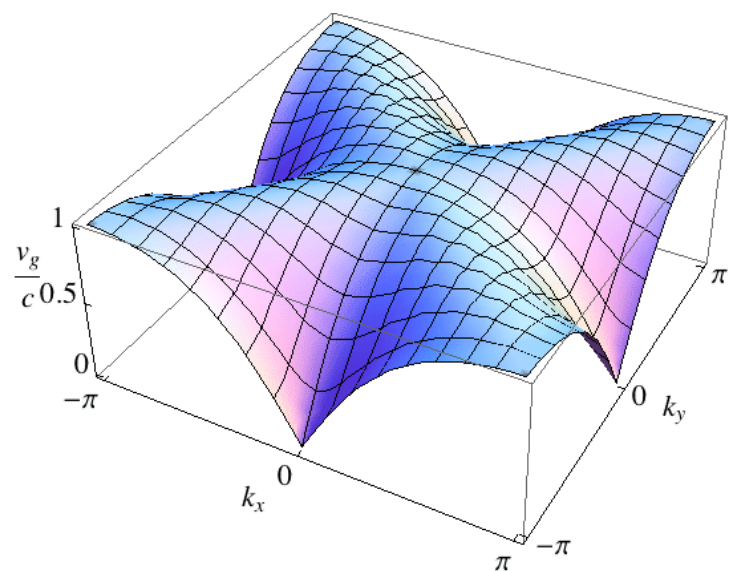

Figure 1. Group velocity $v_{g}$ (normalized to $c$ ) for a zero-mass particle automaton versus the adimensional momentum $\left(k_{x}, k_{y}\right)$ (from Ref. [지]). The speed is approximately isotropic for low momentum (relativistic regime), and becomes anisotropic for very large momenta (ultrarelativistic regime). speed of light in the Dirac equation decreases also versus the mass for very large Planckian masses, the automaton evolution becoming stationary at the Planck mass [E:3], since for larger masses the evolution would be non unitary. The Planck mass is thus the maximum possible mass for a Dirac particle. This conclusion does not come from arguments on the occurrence of a mini black hole, but is just a result of quantum theory.

Energy and momenta are finite in the digital world. The maximum momentum is simply given by the De Broglie relation $\hbar \pi / l_{P}$. For Fermions, we can have only one particle and one antiparticle per Planck cell, and the bound on how much energy can be crammed into a unit of space is determined by the maximum energy per particle, which cannot be more that $\hbar \pi t_{P}^{-1}=6.14663 * 10^{9} \mathrm{~J}$ (a huge energy!). This is the energy for achieving 2 ops [ख]] of the automaton during the Planck time, as given by the Margulus-Levitin theorem [2.5] (each step of the automaton is obtained with two rows of quantum gates). 


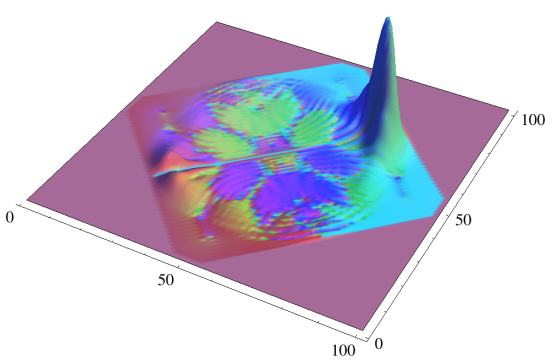

(a)

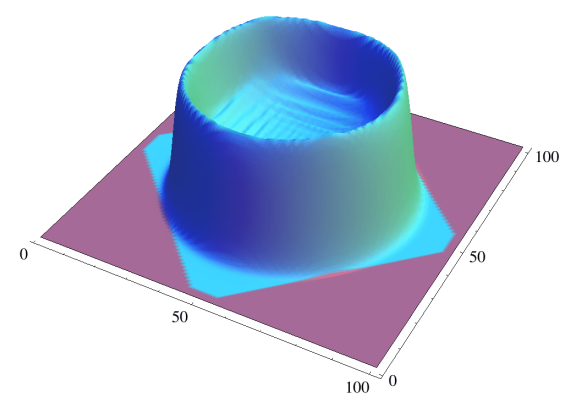

(c)

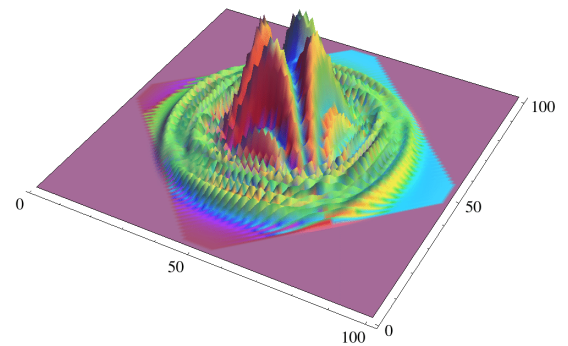

(b)

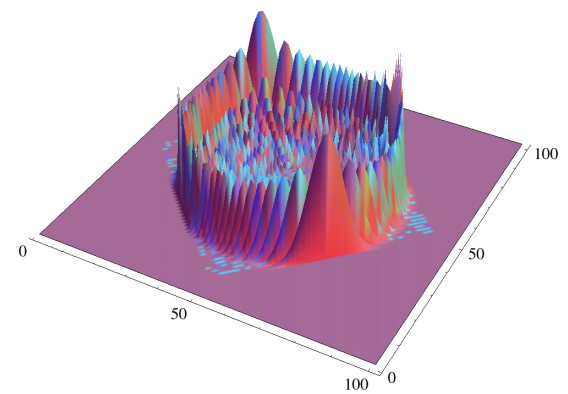

(d)

Figure 2. How particles would look in a digital world made by a quantum automaton: the Dirac automaton for $d=2$ space dimensions. The height of the plot represents the probability of finding a particle with up-spin after 45 Planck times, for different initial states. Colors represents the spin state, and are not relevant for the present purpose. (a,b,c): Gaussian wavepacket with $\Delta_{x}=\Delta_{y}=2 l_{P}$, (d) state localized over a single Planck cell. Fig (a) high relativistic momentum, Fig. (b) ultrarelativistic momentum at Brillouin zone border, Figs. (c,d) zero momentum. (Theory in Ref. [四]).

\section{A quantum-digital space-time}

The quantum nature of the automaton is crucial for the emergence of space-time. There are two crucial points against using a classical automaton.

First point against a classical automaton. With a classical automaton one cannot have isotropic space emerging from an homogeneous classical causal network, due to the Weyl Tile argument [ㅍ]]: we count the same number of tiles in a square lattice both along the diagonal and in the direction of the square sides: where the $\sqrt{2}$ comes from? Indeed, the maximal speed of information in bcc-lattice automaton, as in the Dirac case, would be faster by a factor $\sqrt{2}$ or $\sqrt{3}$ along diagonals than along lattice axes, ending up with an anisotropic space for any homogeneous lattice [ㅈ] , (the problem is not cured by the continuum 
limit). Instead, in a quantum network isotropy is recovered through quantum superpositions of different paths (see e.g. Fig. 『c), and we have again isotropy of max-speed in the relativistic regime of small momenta (Fig. 四), whereas anisotropy would be in principle visible only in the ultrarelativistic regime of huge momenta (Figs. 四, 『b) or for ultra-localized states (Fig. 『d). In a similar manner the quantum nature of the network provides the mechanism for restoration of all continuum symmetries in the relativistic regime. The digital version of Lorentz transformations for a classical homogeneous causal network can be found in Ref. [ए]]: the usual Lorentz covariance cannot be restored from them. Recovering Lorentz covariance from a classical causal network (i.e. describing a causal ordering partial relation) conflicts with the homogeneity principle, and needs a random topology, as in the causal set program of Sorkin [ [सQ]

Second point against a classical automaton. The second reason against classical automata is that quantum superposition of localized states provides a mechanism for directing information in space, by having constant relative phase between neighboring systems in the superposition, thus giving momentum to the information flow. Such mechanism is not possible in a classical causal network. It is the interplay between quantum coherence and nonlocality that plays the crucial role of keeping information going along a desired direction with minimal spreading, a task that cannot be accomplished by a classical automaton.

Emergence of classical mechanics. The Hamiltonian for the classical field theory corresponding to the quantum automaton can be reversely derived from the unitary operator of the automaton [ [2]. Customary quantum particles are Gaussian coherent superposition of single-system states with constant relative phase between neighboring systems, corresponding to the particle momentum: the classical trajectory is the "typical path" along the quantum network, namely the path with maximum probability of the Gaussian packet.

\section{Where is gravity?}

The big question is now where gravity comes from. I still don't have a definite answer, but I believe that the equivalence principle must be rooted in the automaton mechanism, and the gravitational force must emerge at the level of the Dirac free theory, which itself defines the inertial mass. This does not occur in customary quantum field theory, but may happen in the quantum automaton theory, in terms of a tiny "thermodynamic" effect that can occur even for few particles: a purely quantum-digital effect. Indeed, the digital nature of the quantum automaton seems to make it the natural scenario for the generalized holographic principle at the basis of the Jacobson-Verlinde idea of gravity as entropic force [ [], []]. The hypothesis of gravity as a quantum-digital effect is very fascinating: it would mean we are indeed experiencing the quantum-digital nature of the world, in every moment and everywhere: through gravity! 


\section{References}

[1] Feynman and Computation, (Westview Press, Boulder 2002)

[2] G. Chiribella, G. M. D'Ariano, P. Perinotti, Phys. Rev. A 84, 012311 (2011).

[3] C. Brukner, Physics 455 (2011).

[4] L. Hardy, arXiv:1104.2066.

[5] L. Masanes and M. P. Múller, New J. Phys. 13, 063001 (2011).

[6] T. Jacobson, Phys. Rev Lett. 751260 (1995).

[7] E. Verlinde, arXiv: 1001.0785 (2010).

[8] H. Reichenbach, The Philosophy of Space and Time (Dover, New York, 1958)

[9] G. Ludwig and G. Thurler, A New Foundation of Physical Theories, (Springer, New York, Heidelberg, Berlin 2006).

[10] L. Hardy, arXiv:quant-ph/0101012 (2001).

[11] H. Barnum, J. Barrett, L. Orloff Clark, M. Leifer, R. Spekkens, N. Stepanik, A. Wilce, and R. Wilke, New J. Phys. 12, 3024 (2010).

[12] B. Dakic and C. Brukner, arXiv quant-ph, (2009).

[13] B. Coecke, Contemp. Phys. 51, 59 (2010).

[14] B. Russell, Proc. Aristotelian Soc. 131 (1912).

[15] C. A. Fuchs, arXiv:1003.5209 (2010).

[16] L. Hardy and W. K. Wootters, arXiv:1005.4870, (2010).

[17] G. M. D'Ariano, in Philosophy of Quantum Information and Entanglement, edited by A. Bokulich and G. Jaeger (Cambridge University Press, Cambridge, UK, 2010).

[18] D. Deutsch, Royal Soc. Lond. 40097 (1985).

[19] B. Schumacher and R. F. Werner, arXiv:quant-ph/0405174 (2004).

[20] A. Bisio, G. M. D'Ariano, and A. Tosini (unpublished).

[21] G. M. D'Ariano and P. Perinotti (unpublished).

[22] G. M. D'Ariano, FQXi Essay Contest Is Reality Digital or Analog? (2011). See also: G. M. D'Ariano, Il Nuovo Saggiatore 2813 (2012).

[23] G. M. D'Ariano, Phys. Lett. A 376678 (2012) [arxiv:1012.0756].

[24] S. Lloyd, arXiv:1206.6559 (2012).

[25] N. Margolus, and L. B. Levitin, Physica D 120188 (1998).

[26] G. M. D'Ariano and A. Tosini, arXiv:1109.0118 (2011).

[27] H. Weyl, Philosophy of Mathematics and Natural Sciences (Princeton University Press, Princeton) 1949.

[28] T. Fritz, arXiv:1109.1963 (2011).

[29] L. Bombelli, J. H. Lee, D. Meyer, and R. Sorkin, Phys. Rev. Lett 59, 521 (1987) 